\title{
Multi-Drug-Resistant Cells Enriched From Chronic Myeloid Leukemia Cells by Doxorubicin Possess Tumor-Initiating-Cell Properties
}

\author{
Hong Xin ${ }^{1}$, Ying Kong ${ }^{1, \dagger}$, Xiaoxiao Jiang ${ }^{2, \dagger}$, Ke Wang $^{3}$, Xiaoran Qin ${ }^{3}$,Ze-Hong Miao ${ }^{4}$, Yizhun Zhu, ${ }^{1, * a}$, \\ and Wenfu Tan ${ }^{1,4, * b}$ \\ ${ }^{1}$ Department of Pharmacology, School of Pharmacy, Fudan University, Shanghai 201203, P.R. China \\ ${ }^{2}$ Department of Pharmacology, Shandong University at Weihai, Weihai 264209, P.R. China \\ ${ }^{3}$ Department of Pharmacology, Shanghai ChemPartner Co., Ltd., Shanghai 201203, P.R. China \\ ${ }^{4}$ State Key Laboratory of Drug Research, Shanghai Institute of Materia Medica, Chinese Academy of Sciences, \\ Shanghai 201203, P.R. China
}

Received January 24, 2013; Accepted June 7, 2013

\begin{abstract}
Multiple drug resistance (MDR) occurring during chemotherapy is a major obstacle for treatment of cancers using chemotherapeutic drugs; thus, the mechanisms underlying MDR have attracted intensive attention. Many studies have shown that tumor-initiating cells exhibit a chemotherapeutic tolerance characteristic. However, whether the MDR cells possess tumorinitiating cells properties and its underlying mechanisms remain to be fully elucidated. In this study, we utilized a well-established MDR cell line K562/A02 enriched by doxorubicin from $\mathrm{K} 562$ cells to determine if the K562/A02 cells possess tumor-initiating properties and investigated its potential molecular mechanisms. We observed that the expressions of Oct4, Sox2, and Nanog, all of which are well-characterized stem cell markers, in K562/A02 cells were elevated in comparison to parental K562 cells; in addition, we found that K562/A02 cells exhibited more potent in vitro and in vivo tumor-initiating properties, as revealed by sphere assay, self-renewal assay, soft agar assay, and animal studies. Furthermore, our data suggest that snail and twist1, two well known transcriptional factors for the epithelial-mesenchymal transition (EMT) program, may be potentially involved in the acquisition of tumor-initiating properties of K562/A02 cells. Thus, our study demonstrates that MDR K562/A02 cells possess tumor-initiating properties, most likely due to the elevated expressions of snail and twist1.
\end{abstract}

Keywords: multi-drug resistance, K562/A02, tumor-initiating cell, snail, twist1

\section{Introduction}

Although great progresses have been made in "mechanisms-based" anti-cancer therapy, chemotherapy using traditional cytotoxic drugs is still one of the most useful approaches for treatment of cancers, despite of its limitations such as acquired chemoresistance, a major obstacle for successful chemotherapy (1). Acquired drug resistance possesses a property of cross drug resistance, namely, multiple drug resistance (MDR). To combat the

\footnotetext{
${ }^{\dagger}$ These authors contributed equally.

Corresponding author. *azhuyz@fudan.edu.cn, ${ }^{* b}$ wftan@fudan.edu.cn Published online in J-STAGE on August 1, 2013

doi: $10.1254 /$ jphs.13025FP
}

MDR, substantial efforts have been devoted to elucidate the mechanisms underlying the MDR, and a variety of mechanisms have been proposed for interpreting the phenomenon of MDR, ranging from drug efflux pumps, activation of detoxifying enzymes to apoptotic defects, to name a few $(1,2)$.

Tumor-initiating cells, also termed cancer stem cells (CSCs), represent a minor subpopulation of cancer cells with indefinite self-renewal ability, while maintaining their capacity to generate both tumorigenic and nontumorigenic cells $(3,4)$. The tumor-initiating cells theory was initially proposed from acute myeloid leukemia and subsequently extended to solid tumors for a minor subpopulation of cancer cells within a tumor with three features: i) endowed with tumorigenic ability when 
transplanted into immunodeficient mice; ii) characterized by a distinctive profile of surface markers, which can be used to isolate tumorigenic cells from nontumorigenic ones; iii) endowed with ability to generate tumors containing mixed populations of both tumorigenic and nontumorigenic cancers cells $(5-7)$. Recently, studies have shown that tumor-initiating cells can be generated from well-differentiated cancer cells by initiating an epithelial-mesenchymal transition (EMT) program, a critical cellular program for embryonic development and cancer progression. Immortalized human mammary epithelial cells (HMLEs) gain the characteristics of tumor-initiating cells when forced to ectopically express snail or twist1, both of which are well-known transcriptional factors for initiating the EMT program, thus providing a direct link for EMT and CSCs $(8,9)$.

Cytotoxic anti-cancer drugs, which are effective at debulking tumor mass, often fail to eradicate the tumor, thus resulting in the recurrence of cancer. This phenomenon is most likely due to the subset of tumor-initiating cells within the tumor (3). Indeed, accumulating evidences in recent years suggest that the tumor-initiating cells exhibit characteristics of MDR cells, such as elevated expressions of drug efflux proteins and tolerance to chemotherapy drugs, thus suggesting a potential correlation between the tumor-initiating cells and the MDR cells (10). However, whether the MDR cells possess tumor-initiating-cell characteristics and its underlying mechanisms are not fully understood. In this study, we utilized a well-established MDR cell line K562/A02 and its parental cell line K562, a chronic myeloid leukemia cell line, to explore whether MDR cells possess properties of tumor-initiating cells and the potential molecular mechanisms. We found that K562/A02 cells exhibit the properties of tumor-initiating cells and that snail and twist 1 may be potentially responsible for maintaining these properties of MDR cells K562/A02.

\section{Materials and Methods}

\section{Materials}

Rabbit monoclonal antibodies for Oct4, Sox2, and Nanog were purchased from Bioss Corporation (Woburn, MA, USA). Rabbit monoclonal antiserum against snail, twist1, and GAPDH were obtained from Santa Cruz Biotechnology (Santa Cruz, CA, USA). The recombinant human epidermal growth factor (EGF) and basic fibroblast growth factor (bFGF) were obtained from Sigma (St. Louis, MO, USA).

\section{Cell lines}

Chronic myeloid leukemia cell line K562 was obtained from the American Type Culture Collection (Manassas,
VA, USA) and maintained in RPMI 1640 medium (Sigma) containing 10\% fetal calf serum. The doxorubicin selected multi-drug tolerant K562/A02 subline was obtained from the Institute of Hematology, Chinese Academy of Medical Sciences (Tianjin, China), which was routinely maintained in medium containing $200 \mathrm{ng} / \mathrm{ml}$ of doxorubicin (Sigma). The chemoresistant K562/A02 cells were authenticated by comparing their fold resistance with that of the parental cells and examining the expression levels of ABC transporters. K562/A02 cells were cultured in medium without doxorubicin for at least $5-7$ days before they were used for experiments (11).

\section{shRNA and lentivirus infections}

Snail shRNA, twist1shRNA, and shRNA control cloned into the pMAGIC 7.1 lentiviral system were purchased from Sunbio (Shanghai, China). shRNAs and control were transfected to 293T cells using lipofectamine 2000 (Invitrogen, Carlsbad, CA, USA) according to the instructions provided by the manufacturer. Lentiviruses harboring various kinds of shRNA were prepared as previously reported (12).

\section{Sphere formation and self-renewal assay}

Cells were plated into wells of ultra low adherent 96-well plate (Corning, Lowell, MA, USA) at 500 cells/well in DMEM/F12 (Invitrogen) supplemented with B27, $2 \mathrm{mM}$ glutamine (Invitrogen), $10 \mathrm{ng} / \mathrm{ml} \mathrm{EGF}$, $10 \mathrm{ng} / \mathrm{ml} \mathrm{bFGF,} 0.5 \mathrm{mg} / \mathrm{ml}$ hydrocortisone (Sigma), and $10 \mu \mathrm{g} / \mathrm{ml}$ insulin (Sigma). Fresh medium was added every $3-4$ days. After 15 days, sphere numbers were determined under a microscope and the size of the spheres in terms of their diameters was measured using NIH image J. For the self-renewal assay, the spheres were collected, dissociated, and re-plated into wells of an ultra low adherent 96-well plate. The number and size of spheres were determined as mentioned above after culturing under the same conditions for 15 days.

\section{Soft agar assay}

Cells were harvested and suspended in culture medium. To make the bottom layer, $1 \mathrm{ml}$ of $0.5 \%$ agar (Invitrogen) was added to 6-well plates and allowed to gel at room temperature. To prepare the top layer $(0.25 \%$ agar $), 500 \mu \mathrm{l}$ of $0.5 \%$ agar was mixed with $500 \mu$ cell suspension containing 5000 cells. This mixture was overlaid above the bottom layer and allowed to solidify at room temperature. An additional $2 \mathrm{ml}$ of culture medium was added after solidification to the top layer, followed by routine incubation for 2 weeks. After that, the colonies were photographed. The colony numbers were indirectly reflected through measuring the cell numbers with 
FlexStation 3 (Excitation 530 nm, Emission 590 nm; Molecular Devices, Sunnyvale, CA, USA) after Alamar Blue dye (Invitrogen) staining.

Reverse transcription and quantitative PCR (QT-PCR) analyses

Total RNA was isolated with the Trizol reagent (TaKaRa, Dalian, China) according to the instructions provided by the manufacturer. Total RNA was reversely transcribed using Superscript ${ }^{\mathrm{TM}}$ III reverse transcriptase. Then the cDNAs were amplified by Real-time PCR (iQ5; Bio-Rad, Hercules, CA, USA) with the SYBR-Green kit (TaKaRa) using the following primers (Invitrogen, Shanghai, China): Oct4: 5'-ATTCAGCCAAACGACCA TCT-3', 5'-TTGCCTCTCCACTCGGTTCTC-3'; Sox2: 5'-ACACCAATCCCATCCACACT-3', 5'-GCAAAC TTCCTGCAAAGCTC-3'; Nanog: 5'-GTCCCGGTC AAGAAACAGAA-3', 5'-TGCGTCACACCATTGCT ATT-3'; Snail: 5'-GAGGCGGTGGCAGACTAG-3', 5'GACACATCGGTCAGACCAG-3'; twist 1: 5'-GGAGTC CGCAGTCTTACGAG-3', 5'-TCTGGAGGACCTGGT AGAGG-3'; TATA: 5'-ACCCTTCACCAATGACTCCT ATG-3', 5'-TGACTGCAGCAAATCGCTTGG-3'. The alteration of mRNA expression in cells was assessed using the iQ5 optical system software by delta delta $\mathrm{Ct}$ method.

\section{Immunoblot analysis}

Cells were lysed in lysis buffer $(50 \mathrm{mM}$ Tris- $\mathrm{HCl}$, $150 \mathrm{mM} \mathrm{NaCl}, 1 \%$ Nonidet P-40) supplemented with protease inhibitors $(0.5 \mathrm{mM}$ phenylmethylsulfonyl fluoride, $1 \mu \mathrm{g} / \mathrm{ml}$ aprotinin, and leupeptin) for $15 \mathrm{~min}$ on ice. Equal amounts of protein were subjected to SDSpolyacrylamide gel electrophoresis and transferred onto a polyvinylidene difluoride membrane (Immobilon P; Millipore, Billerica, MA, USA). The membranes were then incubated with the appropriate antibodies as indicated.

\section{Tumorigenic assays}

Aliquots of $10^{3}$ and $10^{2} \mathrm{~K} 562$ or K562/A02 cells mixed with matrigel (BD, San Jose, CA) were injected subcutaneously into NOD-SCID mice (Vital River, Beijing, China). The tumor incidence was monitored for 60 days following injection. All procedures were preapproved by the Animal Care and Use Committee of the Fudan University and performed according to institutional policies.

\section{Statistical analysis}

Statistical analysis of QT-PCR and self-renewal analysis were performed by Student's $t$-test, and in vivo tumorigenic assay was performed by the MantelHaenszel test. Asterisks denote statistic significance $\left({ }^{*} P<0.05,{ }^{* *} P<0.01\right)$.

\section{Results}

K562/A02 cells exhibit higher expression of stem cell markers in comparison to parental K562 cells

To determine the tumor-initiating cells properties of K562/A02 cells, we first examined the expressions of pluripotency stem cell markers, such as Oct4, Sox2, and Nanog, which are often found in poorly differentiated tumors and recognized as markers of tumor-initiating cells $(9,13)$. QT-PCR analyses showed that K562/A02 cells exhibited higher expression of Oct4, Sox2, and Nanog at mRNA level when compared to parental K562 cells (Fig. 1A). Concomitantly, we observed that the protein level expressions of Oct4, Sox2, and Nanog in K562/A02 cells were all elevated with respect to parental K562 cells (Fig. 1B).

K562/A02 cells exhibit more potent sphere formation ability and self-renewal capacity compared to K562 cells

Formation of spherical cellular aggregates is another key feature of tumor-initiating cells (14). To compare the
A

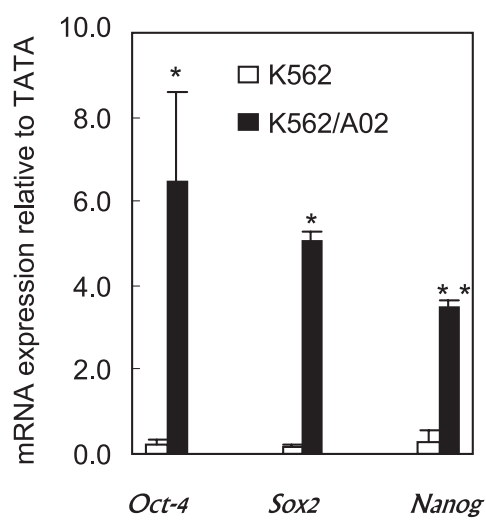

B

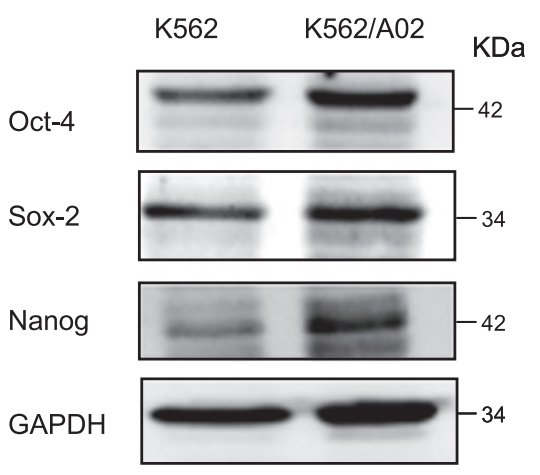

Fig. 1. The expressions of stem cell markers Oct4, Sox 2 and Nanog in K562 and K562/A02 cells. A: QTPCR analysis of the expressions of Oct4, Sox2, and Nanog in K562 and K562/A02 cells. Total RNA was extracted and used for reverse transcription and QTPCR analysis as mentioned in Materials and Methods. Data are expressed as the average \pm S.D. of results obtained from three separate experiments. B: Western blot analysis of the expressions of Oct4, Sox2, and Nanog in K562 and K562/A02 cells. Cells were lysed and used for western blotting analysis as mentioned in materials and methods, using the GAPDH as a loading control. Blots are representative of three independent experiments. 
A

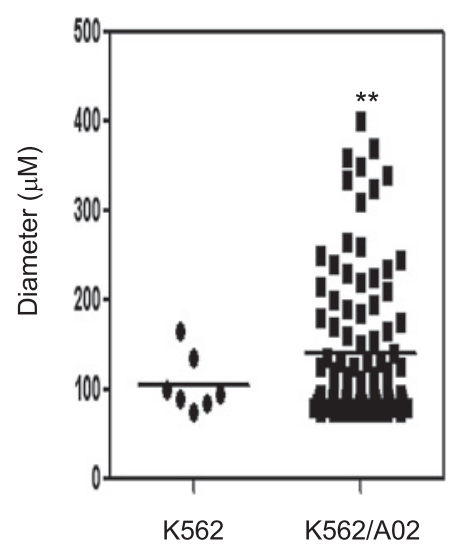

B

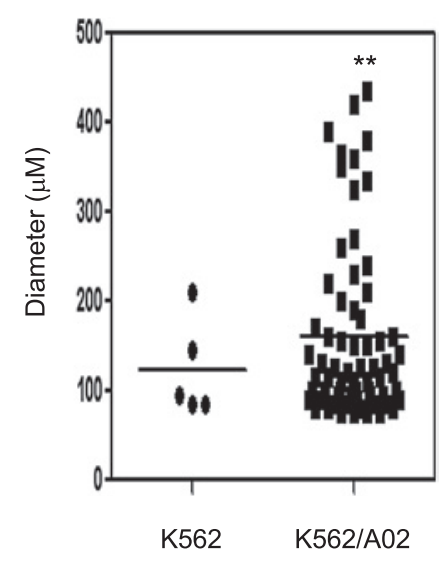

Fig. 2. Sphere formation and self-renewal abilities of K562 cells and K562/A02 cells. A: Scatter plot of the diameters of spheres formed by K562 cells and K562/A02 cells (1st generation). B: Scatter plot of the diameters of spheres formed by K562 cells and K562/A02 cells (2nd generation).

A

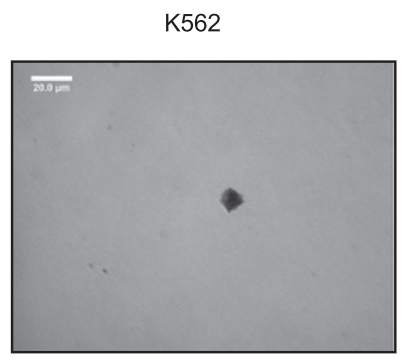

K562/A02

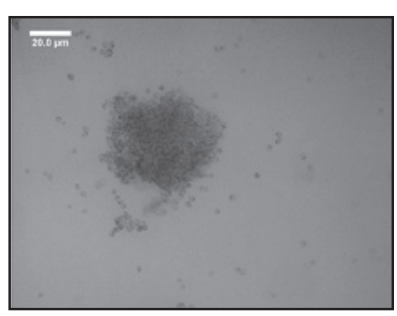

B

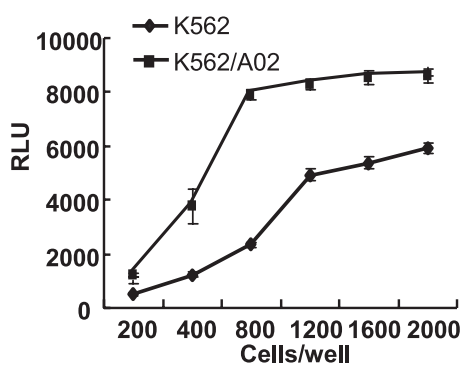

Fig. 3. K562/A02 cells possess in vitro tumor-initiating ability compared to K562 cells. A Typical colonies formed by K562 cells and K562/A02 cells. Scale bar: $20.0 \mu \mathrm{m}$. B: Colony numbers formed by K562 and K562/A02 cells were determined by the cell numbers.

sphere formation ability of K562 and K562/A02 cells, we cultivated the cells using ultra low adhesion plates that favor the proliferation of undifferentiated cells in serum free medium containing EGF and bFGF. After about 15-days culture in such conditions, we observed that K562/A02 cells formed much more sphere-like clusters than K562 cells, with cluster numbers of 13 and 136 for K562 cells and K562/A02 cells, respectively (Fig. 2A). Meanwhile, the sphere-like clusters developed from K562/A02 cells were much larger than those that developed from K562 cells, with the average size of $117.2 \pm 6.5 \mu \mathrm{M}$ for $\mathrm{K} 562 / \mathrm{A} 02$ cells and $86.9 \pm 8.8 \mu \mathrm{M}$ for K562 cells, respectively (Fig. 2A). These observations support that K562/A02 cells exhibit more potent sphere formation capacity compared to K562 cells. We further dissociated the spheres formed by K562/A02 cells and K562 cells into single cells to generate secondary generation of spheres to measure the selfrenewal capability, which represents an ability of cancer stem cells to form new stem cells with identical, intact potential for proliferation, expansion, and differentiation, thus maintaining the stem cell pool (15). We observed the K562/A02 cells still formed much more and larger sphere clusters, with the number of 87 and average diameter of $136.7 \pm 10.2 \mu \mathrm{M}$, whereas $\mathrm{K} 562$ cells formed sphere clusters with the number of 16 and average diameter of $87 \mu \mathrm{M}$ (Fig. 2B). These studies show that K562/A02 cells display more potent sphere formation and self-renewal ability with respect to K562 cells.

\section{K562/A02 cells possess in vitro tumor-initiating ability compared to K562 cells}

To determine whether K562/A02 cells possess tumorinitiating properties, we tested the cologenic capacity of K562/A02 and K562 cells by soft agar assay, a widely used in vitro tumorigenic assay (16). After around 15 days cultured in such an anchorage-independent situation, the K562/A02 cells formed much larger colonies compared to K562 cells (Fig. 3A). The cell numbers, which were used to indirectly reflect the colony numbers, in K562/A02 were much more than those in K562 cells (Fig. 3B). These observations suggest that K562/A02 cells exhibit more potent in vitro tumorigenic capacity in comparison to K562 cells. 
Table 1. Tumor incidence of K562 cells and K562/A02 cells injected into NOD-SCID mice in limiting dilutions

\begin{tabular}{lcc}
\hline & Numbers of injections / Tumors incidence \\
\cline { 2 - 3 } Cells injected & $1 \times 10^{3}$ & $1 \times 10^{2}$ \\
\hline K562 & $10 / 0$ & $10 / 0$ \\
K562/A02 $(* *)$ & $10 / 4$ & $10 / 2$ \\
\hline
\end{tabular}

K562/A02 cells possess in vivo tumor-initiating ability compared to K562 cells

To further strengthen the notion that K562/A02 cells possess tumorigenic potential, we xenografted subcutaneously different numbers $\left(10^{3}, 10^{2}\right)$ of K562/A02 cells or K562 cells mixed with matrigel into NOD-SCID mice to assess the in vivo tumorigenic ability of $\mathrm{K} 562 /$ A02 and K562 cells. The result showed that when $10^{2}$ or $10^{3}$ cells were implanted into NOD-SCID mice, K562 cells failed to generate tumors, whereas we observed that there were 2 , and 4 tumors developed for injections of $10^{2}$ and $10^{3} \mathrm{~K} 562 / \mathrm{A} 02$ cells, respectively $(\mathrm{n}=10)$, as shown in Table 1. These observations further strongly support that K562/A02 cells possess tumorigenic potential in vivo.

Snail and twist 1 are both potentially involved in maintaining the tumor-initiating properties of K562/A02 cells

Transcriptional factors involved in EMT such as snail or twist 1 may endow more differentiated cancer cells with properties of tumor-initiating cells $(8,9)$. We then asked whether transcriptional factors involved in EMT could account for the tumor-initiating-cell phenotype of K562/A02 cells. As anticipated, we found that the expression of snail and twist 1 at the mRNA and protein level were both elevated in K562/A02 cells relative to parental K562 cells, as revealed by QT-PCR (Fig. 4A) and western blotting analysis (Fig. 4B). Furthermore, we observed that knocking-down the expressions of snail and twist 1 in K562/A02 cells (Fig. 4C) reduced the expressions of stem cell markers such as Oct4, Sox2, and Nanog (Fig. 4D). These results indicate that both snail and twist 1 may be potentially involved in keeping the tumorigenic properties of K562/A02 cells.

\section{Discussion}

Tumor-initiating cells, also termed cancer stem cells, are defined by their indefinitely self-renewal ability, while maintaining their capacity to generate both tumorigenic and non-tumorigenic cells. This kind of cells has been shown to possess the phenotype of chemoresistance, as reflected by enhanced ability for DNA repair, decreased apoptosis, and elevated expressions of ATPbinding cassette transporters (10). In the present study, using a well-established MDR cells enriched from chronic myeloid leukemia cells K562 by doxorubicin, we show that MDR K562/A02 cells possess tumorinitiating-cell properties, including elevated stemness signature markers Oct4, Sox2, and Nanog, increased sphere formation and self-renewal abilities, enhanced clonogenic ability, and in vivo tumor-initiating capacity. These data demonstrate that K562/A02 cells exhibit tumor-initiating-cell features. Meanwhile, our data show
A

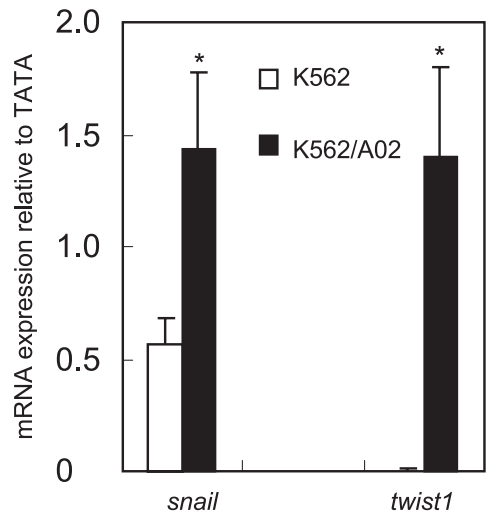

C

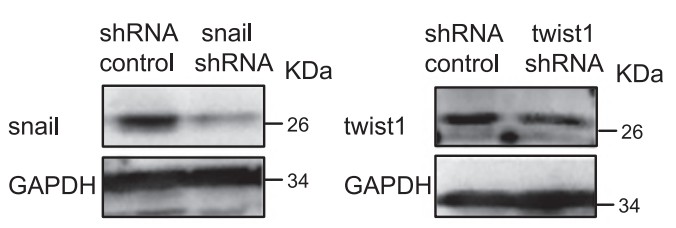

B

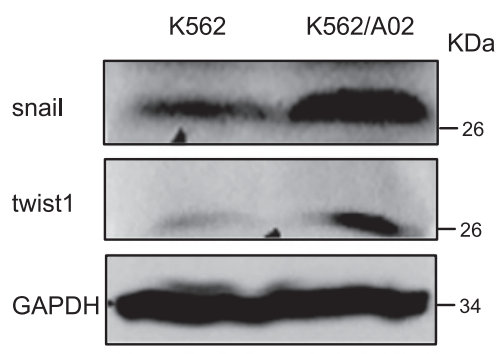

D

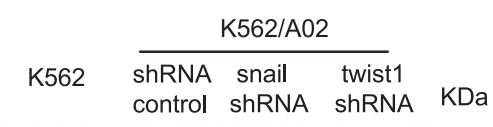

Oct4

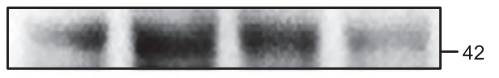

Sox-2

Nanog

GAPDH
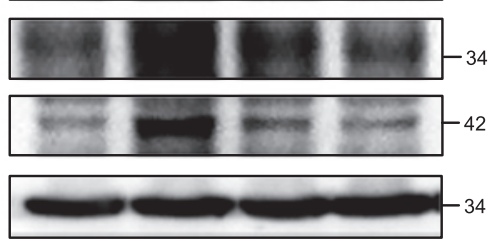

Fig. 4. Snail and twistl are potentially involved in maintaining the tumor-initiating cells properties of K562/A02 cells. A: The transcripts of snail and twist 1 in K562 and K562/A02 cells were measured by QT-PCR assay. B: Western blot analysis of the protein expressions of snail and twist1 in $\mathrm{K} 562$ and $\mathrm{K} 562 / \mathrm{A} 02$ cells. Blots are representative of three independent experiments. C: Western blot analysis of the expressions of snail and twist1 in K562/A02 cells infected by lentiviruses carrying snail shRNA, twist1 shRNA, or shRNA control. D: Knockdown of the expressions of snail and twist 1 reduced expressions of Oct4, Sox2, and Nanog. 
that K562/A02 cells exhibit higher expressions of EMT transcriptional factors snail and twist 1 than parental K562 cells, suggesting that both snail and twist 1 may be involved in the properties of K562/A02 cells.

Weinberg and colleagues have recently shown that forced expression of transcriptional factors involved in EMT such as snail or twist1 to neoplastic mammary epithelial cells could generate tumor-initiating cells, thus providing a direct link between transcriptional factors such as snail or twist1 and properties of tumor-initiating cells $(8,9)$. In the current work, we found that the expressions of snail and twist 1 were both elevated in K562/A02 cells when compared to the parental K562 cells, and knockdown of the expressions of snail and twist1 decreased the expressions of stem cell markers such as Oct4, Sox2, and Nanog. These observations indicate that snail and twist1 are both involved in maintaining the tumor-initiating properties of MDR K562/A02 cells. Indeed, we further observed that limiting the expression of snail or twist 1 in K562/A02 cells abolishes the tumorinitiating properties of K562/A02 cells, including sphere formation and self-renewal ability (data to be published). Thus, we suggest that snail and twist 1 may be involved in maintaining the tumor-initiating-cell properties of MDR cells K562/A02.

It is becoming evident that chemotherapy fails to eliminate the tumor-initiating cells in the tumor bulk, therefore leading to relapse of tumors and consequently chemo-resistance. Our present study demonstrates that multi-drug-resistant cells enriched from chronic myeloid leukemia cells by doxorubicin exhibit tumor-initiatingcell properties. Similar conclusions were also achieved in MDR cells developed from breast cancer, colon cancer, and lung cancer $(17-19)$. Hence, our present study together with other reports suggests that therapy targeting tumor-initiating cells represents a promising and potential avenue for circumventing chemoresistance.

\section{Acknowledgments}

This work was financially supported by National Natural Science Foundation of China (81173077), the "Interdisciplinary Cooperation Team" Program for Science and Technology Innovation of the Chinese Academy of Sciences, and State Key Laboratory of Drug Research of Chinese Academy of Sciences (SIMM1004KF-07).

\section{Conflicts of Interest}

The authors have no conflicts of interest to disclose.

\section{References}

1 Baguley BC. Multiple drug resistance mechanisms in cancer. Mol Biotechnol. 2010;46:308-316.
2 Ozben T. Mechanisms and strategies to overcome multiple drug resistance in cancer. FEBS Lett. 2006;580:2903-2909.

3 Reya T, Morrison SJ, Clarke MF, Weissman IL. Stem cells, cancer, and cancer stem cells. Nature. 2001;414:105-111.

4 Clarke MF, Dick JE, Dirks PB, Eaves CJ, Jamieson CH, Jones DL, et al. Cancer stem cells--perspectives on current status and future directions: AACR Workshop on cancer stem cells. Cancer Res. 2006;66:9339-9344.

5 Al-Hajj M, Wicha MS, Benito-Hernandez A, Morrison SJ, Clarke MF. Prospective identification of tumorigenic breast cancer cells. Proc Natl Acad Sci U S A. 2003;100:3983-3988.

6 Schatton T, Murphy GF, Frank NY, Yamaura K, Waaga-Gasser AM, Gasser M, et al. Identification of cells initiating human melanomas. Nature. 2008;451:345-349.

7 Bonnet D, Dick JE. Human acute myeloid leukemia is organized as a hierarchy that originates from a primitive hematopoietic cell. Nat Med. 1997;3:730-737.

8 Mani SA, Guo W, Liao MJ, Eaton EN, Ayyanan A, Zhou AY, et al. The epithelial-mesenchymal transition generates cells with properties of stem cells. Cell. 2008;133:704-715.

9 Kong D, Banerjee S, Ahmad A, Li Y, Wang Z, Sethi S, et al. Epithelial to mesenchymal transition is mechanistically linked with stem cell signatures in prostate cancer cells. PLoS One. 2010;5:e12445.

10 Dean M, Fojo T, Bates S. Tumour stem cells and drug resistance. Nat Rev Cancer. 2005;5:275-284.

11 Luan FJ. [Establishment of the multidrug-resistant cell line K562/A02 and its drug-resistant properties]. Zhonghua Zhong Liu Za Zhi. 1993;15:101-103. (text in Chinese with English abstract)

12 Tan W, Martin D, Gutkind JS. The Galpha13-Rho signaling axis is required for SDF-1-induced migration through CXCR4. J Biol Chem. 2006;281:39542-39549.

13 Ben-Porath I, Thomson MW, Carey VJ, Ge R, Bell GW, Regev A, et al. An embryonic stem cell-like gene expression signature in poorly differentiated aggressive human tumors. Nat Genet. 2008;40:499-507.

14 Ponti D, Costa A, Zaffaroni N, Pratesi G, Petrangolini G, Coradini $\mathrm{D}$, et al. Isolation and in vitro propagation of tumorigenic breast cancer cells with stem/progenitor cell properties. Cancer Res. 2005;65:5506-5511.

15 Dalerba P, Cho RW, Clarke MF. Cancer stem cells: models and concepts. Annu Rev Med. 2007;58:267-284.

16 Shin SI, Freedman VH, Risser R, Pollack R. Tumorigenicity of virus-transformed cells in nude mice is correlated specifically with anchorage independent growth in vitro. Proc Natl Acad Sci U S A. 1975;72:4435-4439.

17 Calcagno AM, Salcido CD, Gillet JP, Wu CP, Fostel JM, Mumau $\mathrm{MD}$, et al. Prolonged drug selection of breast cancer cells and enrichment of cancer stem cell characteristics. J Natl Cancer Inst. 2010;102:1637-1652.

18 Dylla SJ, Beviglia L, Park IK, Chartier C, Raval J, Ngan L, et al. Colorectal cancer stem cells are enriched in xenogeneic tumors following chemotherapy. PLoS One; 2008;3:e2428.

19 Levina V, Marrangoni AM, DeMarco R, Gorelik E, Lokshin AE. Drug-selected human lung cancer stem cells: cytokine network, tumorigenic and metastatic properties. PLoS One. 2008;3:e3077. 